\title{
CAPSULAR COMPLIANCE AND PRESSURE-VOLUME RELATIONSHIPS IN NORMAL AND ARTHRITIC KNEES
}

\author{
D. B. Myers and D. G. Palmer, Dunedin, New Zealand
}

From the Rheumatic Diseases Unit, Wellcome Medical Research Institute, Department of Medicine, University of Otago Medical School, Dunedin

This study* of joint capsular compliance was undertaken in an attempt to assess objectively the effect of rheumatoid arthritis on the integrity of the joint capsule and associated ligaments. Joint instability and subluxation in progressive rheumatoid disease may result from damage to these structures through granulomatous invasion and distension by intra-articular effusion. Alteration in the compliance of the joint capsule and associated ligaments might be important as being early evidence of significant tissue damage.

Changes in the properties of joint tissues which can be related to joint symptoms are of theoretical importance. Urayama (1956) investigated the relationship between pressure and volume after saline was injected into the knee joints of 162 patients and normal volunteers. He found significant differences between normal joints and those joints with symptoms arising from injury or disease. Jayson and Dixon (1970a, $b, c)$ confirmed Urayama's findings (1956) of altered pressure-volume relationships in knee joints of patients with rheumatoid arthritis, and discussed the possible pathological effects of persistently increased intra-articular pressures.

In the present study the observations of Urayama (1956) and of Jayson and Dixon $(1970 a, b, c)$ have been extended and the results published by these authors compared with our own. Correlations with clinical information were made when possible. Some kinetic observations on fluid movement in and out of the human knee joint were also obtained.

\section{METHODS}

The methods and apparatus used to measure the hydrostatic intra-articular pressure and the intra-articular volume were described previously (Palmer and Myers 1968). Three young healthy volunteers with no history of joint disability were used as controls. Diagnosis of rheumatoid arthritis fulfilled the definite or classical criteria of Ropes (1959). The three knees affected by osteoarthritis were selected because of the presence of a low pressure effusion requiring aspiration.

Intra-articular hydrostatic pressure was measured with a Statham B transducer connected through a two-way tap to a syringe and needle. Intra-articular volume was determined from the total amount of fluid which was instilled or withdrawn from a joint. Measurements were made in several different sequences. In the case of rheumatoid joints which contained fluid under pressure, recordings were made during step-wise withdrawal of the effusion fluid. In other cases the relationship between intra-articular volume and pressure was determined during both step-wise injection of normal saline into the empty joint and during subsequent step-wise withdrawal of saline from the joint. In a few cases, saline instilled to a pressure of 50 millimetres of mercury was left in the joint and the intra-articular pressure recorded periodically over a period of two hours. The residual fluid was then removed and the volume absorbed over this period determined. The pressure-volume observations were plotted graphically and the compliance $\mathrm{dV} / \mathrm{dP}$ (that is, the change in intra-articular volume per unit change in intraarticular pressure) determined at several levels of intra-articular pressure.

\footnotetext{
A preliminary presentation of the findings described in this paper was made to the annual meeting of the New Zealand Rheumatism Association, February 1970. An abstract of this presentation has been published (Myers and Palmer 1970). 
Clinical information which was recorded included age, sex, period of joint involvement and the period elapsed since any corticosteroid preparation was last injected into the joint.

\section{RESULTS}

Clinical information is presented in Table I.

Pressure-volume curves- $A$ pressure-volume curve typical of those obtained during the instillation and withdrawal of saline from the knee joints of healthy volunteers is shown in

TABLE I

Clinical Detail.s in Patients and Healthy Volunteers

\begin{tabular}{|c|c|c|c|c|c|c|}
\hline $\begin{array}{c}\text { Case } \\
\text { number }\end{array}$ & Disorder & $\begin{array}{c}\text { Age } \\
\text { (years) }\end{array}$ & Sex & $\begin{array}{c}\text { Knee } \\
\text { involved }\end{array}$ & $\begin{array}{c}\text { Period of } \\
\text { involvement } \\
\quad \text { (years) }\end{array}$ & $\begin{array}{c}\text { Period } \\
\text { since local } \\
\text { steroid } \\
\text { (months) }\end{array}$ \\
\hline 1 & None & 21 & Male & Right & - & - \\
\hline 2 & None & 23 & Male & Right & - & $\ldots$ \\
\hline 3 & None & 23 & Male & Right & - & $\ldots$ \\
\hline 4 & Osteoarthritis & 66 & Female & Right & 10 & - \\
\hline 5 & Osteoarthritis & 51 & Female & Left & 2 & - \\
\hline 6 & Osteoarthritis & 74 & Female & Right & 6 & 5 \\
\hline 7 & Rheumatoid arthritis & 23 & Female & Right & $1 \cdot 3$ & 4 \\
\hline 8 & Rheumatoid arthritis & 27 & Male & Left & 3 & 3 \\
\hline 9 & Rheumatoid arthritis & 33 & Female & Right and left & 10 & - \\
\hline 10 & Rheumatoid arthritis & 41 & Male & Right & 2 & 2 \\
\hline 11 & Rheumatoid arthritis & 42 & Female & Right & 4 & 1 \\
\hline 12 & Rheumatoid arthritis & 53 & Female & Left & 22 & 12 \\
\hline 13 & Rheumatoid arthritis & 56 & Female & Right & 4 & 3 \\
\hline 14 & Rheumatoid arthritis & 56 & Male & Right & 2 & - \\
\hline 15 & Rheumatoid arthritis & 56 & Female & Left & 12 & 9 \\
\hline 16 & Rheumatoid arthritis & 61 & Male & Left & 7 & 9 \\
\hline 17 & Rheumatoid arthritis & 62 & Female & Right and left & 10 & - \\
\hline 18 & Rheumatoid arthritis & 65 & Male & Right & $0 \cdot 7$ & - \\
\hline 19 & Rheumatoid arthritis & 66 & Female & Right and left & 3 & 24 \\
\hline 20 & Rheumatoid arthritis & 66 & Male & Right & 20 & 2 \\
\hline
\end{tabular}

Figure I (Case $\left.2^{*}\right)$. The intra-articular pressure increased gradually at low volumes, reaching a pressure of 5 to 10 millimetres of mercury at a volume of 50 cubic centimetres. The pressure then rose more steeply, approaching 50 millimetres of mercury at a volume of 100 cubic centimetres. The pressure-volume curves obtained for patients under the age of thirty-five years with rheumatoid arthritis (examples are given in Figures 1 and 2, Cases 7, 8 and 9) were very similar to the normal type of curve. A pressure-volume curve typical of those obtained for the

* The case numbers of subjects have been recorded to allow cross-reference to the table.

VOL. 54 B, NO. 4, NOVFMBER 1972 
three patients with osteoarthritis is also given in Figure 1 (Case 6). The pressure-volume relationships for these patients were also quite similar to those for the normal controls. However, in patients over the age of forty years with rheumatoid arthritis, the intra-articular pressures rose steeply even at low pressures, approaching 50 millimetres of mercury at a volume of 50 cubic centimetres (Fig. 2, Cases 10,11 and 14).

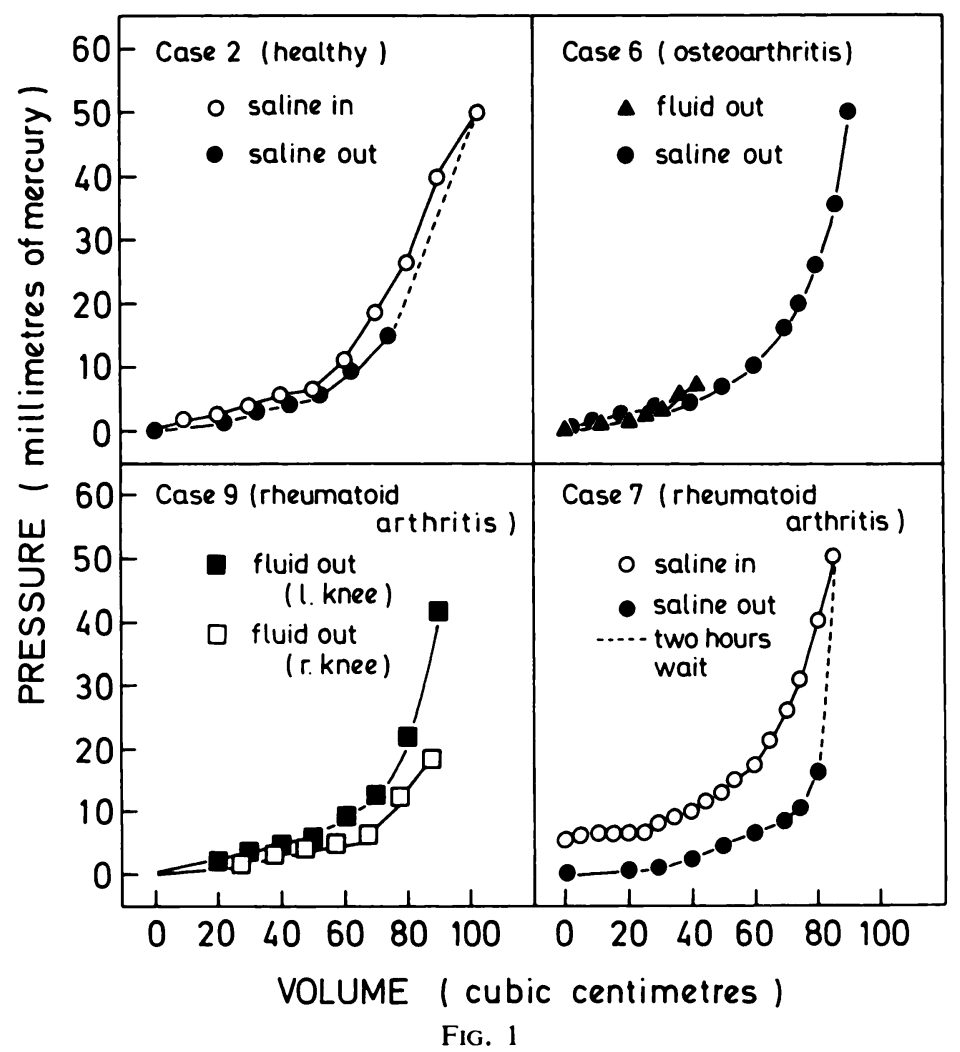

Pressure-volume curves for human knee joints. Typical curves are shown for healthy volunteers (Case 2), patients with osteoarthritis (Case 6), and patients with rheumatoid arthritis under the age of 35 (Cases 7 and 9). Note the general similarities of the curves.

Pressure-volume curves obtained by more than one of the three methods used (withdrawal of an effusion or of saline, or instillation of saline) were similar for the same joint (Fig. 2, Case 8). However, the curves obtained during saline withdrawal were generally displaced slightly to the right of those obtained during saline instillation (Figs. 1 and 2), indicating a small change in compliance as a result of distension.

In two patients with rheumatoid arthritis (both under thirty-five) both knee joints of the same patient gave similar pressure-volume curves. In one case the same joint was studied on two separate occasions three months apart and the curves obtained on each occasion were similar. In those cases in which an effusion was present the point representing the initial pressure and volume of the effusion fell on or very close to the pressure-volume curve subsequently determined with saline (Figs. 1 and 2). The "recycling" of saline (rapid withdrawal and replacement) at a pressure of about 20 millimetres of mercury apparently had no significant effect on the shape of the pressure-volume curve.

Pressure-time curves-Saline was instilled to an initial pressure of 50 millimetres of mercury into the knee joints of one normal volunteer and four patients with rheumatoid arthritis (who 
were over the age of forty years) and the pressure was then recorded at intervals for two hours. In all cases the pressure fell fairly rapidly for the first twenty minutes at a rate of about 1 millimetre per minute, then more gradually for the remaining period at a rate of about $0 \cdot 1$ millimetre of mercury per minute (Fig. 3). The fall in fluid volume over the two-hour period averaged 10 millilitres.

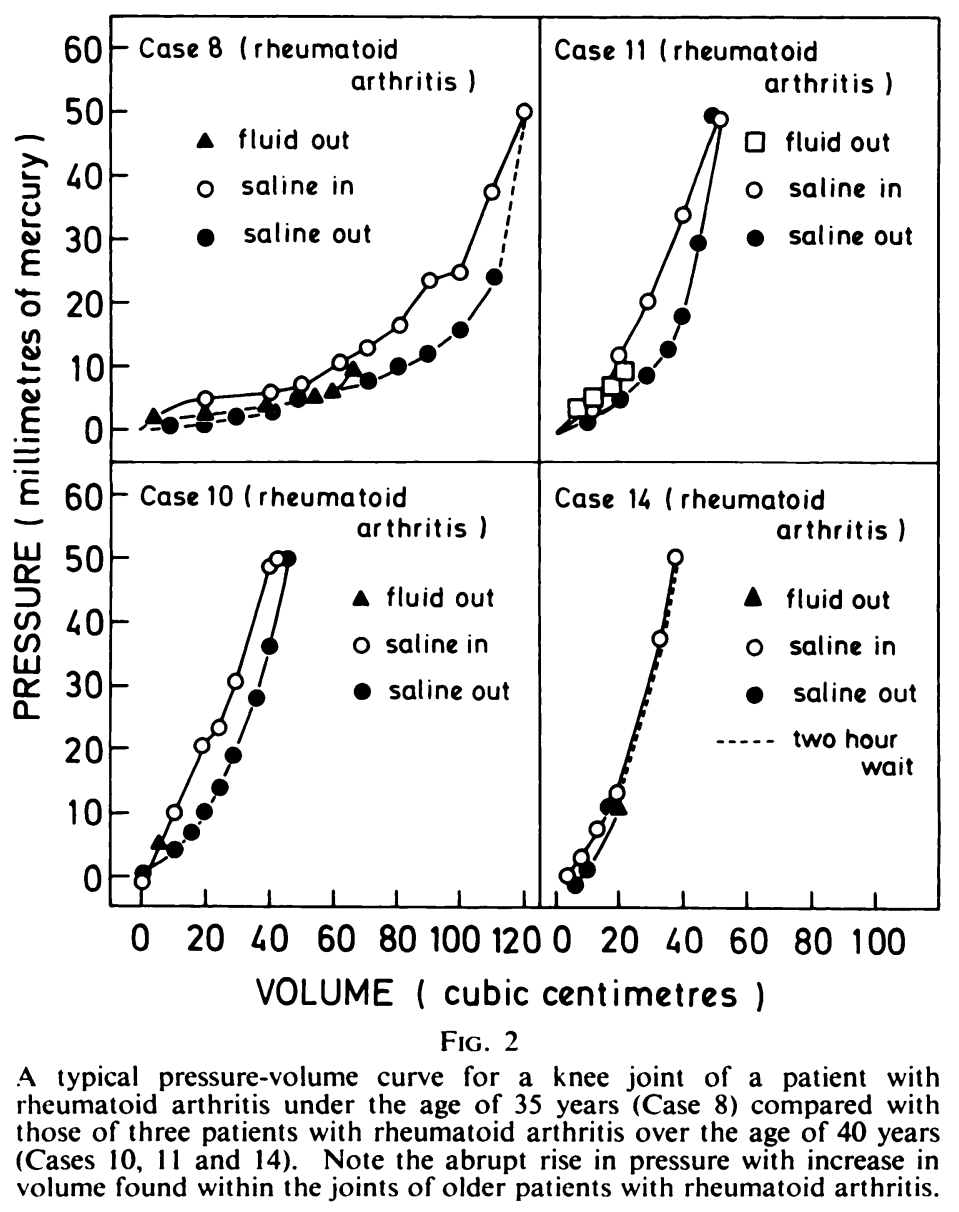

Compliance--The average joint compliance $(\mathrm{dV} / \mathrm{dP})$ for normal volunteers, patients with osteoarthritis and patients with rheumatoid arthritis at low, moderate and high intra-articular pressures $(5,10,15$ and 50 millimetres of mercury respectively) are given in Table II. The results for patients over the age of forty years with rheumatoid arthritis are averaged separately from those for patients under thirty-five years. Compliance values estimated from pressurevolume findings published by Urayama (1956) and Jayson and Dixon (1970a) are included for comparison.

No correlation was found between individual joint compliances and any of the patient variables given in Table I, other than that between compliance and age group (under thirty-five or over forty) for patients with rheumatoid arthritis.

\section{DISCUSSION}

This study has confirmed previous reports (Urayama 1956, Jayson and Dixon 1970a, b, c) that measurements of the pressure-volume relationship in human joints can be useful in assessing the effects of joint distension on capsular tissues. Normal joints contain only very

VOL. 54 B, NO. 4, NOVEMBER 1972 
small fluid volumes, and are subjected to low, sometimes negative, intra-articular pressure at rest (Jayson and Dixon 1970a). Inflamed joints, however, often contain large volumes of fluid under relatively high pressure even at rest (Palmer and Myers 1968). It is informative

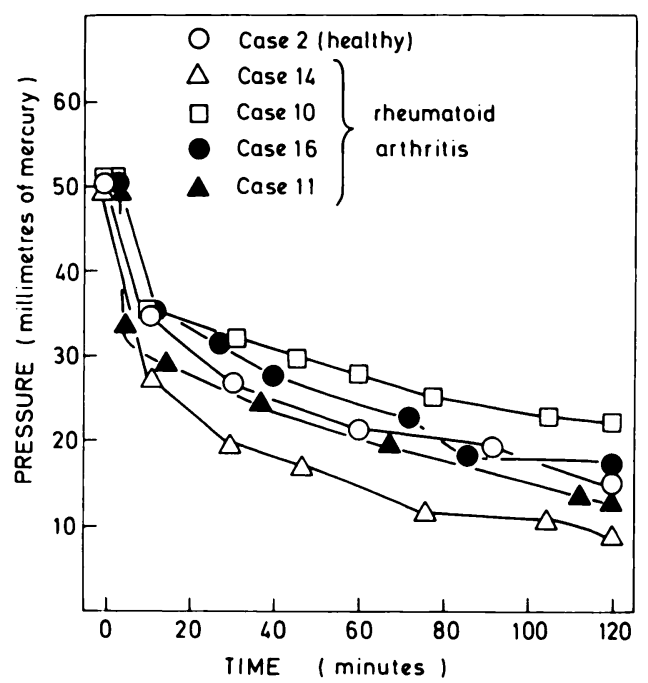

FIG. 3

Pressure-time curves for knee joints of a healthy volunteer (Case 2) and patients with rheumatoid arthritis (Cases 10,11, 14 and 16), which had been injected with normal saline to a pressure of 50 millimetres of mercury. The fall in pressure was greater than could be accounted for by resorption of fluid from the joint cavity in all cases.

TABLE II

Average Estimated Values of Joint Compliance

\begin{tabular}{|c|c|c|c|c|c|c|c|}
\hline \multirow{3}{*}{ Study } & \multirow{3}{*}{ Disease } & \multirow{3}{*}{\multicolumn{2}{|c|}{ Number of joints }} & \multicolumn{4}{|c|}{ Intra-articular pressure (millimetres $\mathrm{Hg}$ ) } \\
\hline & & & & & & & \\
\hline & & & & & $\begin{array}{l}\text { verag } \\
\text { ntime }\end{array}$ & $\begin{array}{l}\text { plian } \\
\text { illim }\end{array}$ & \\
\hline \multirow{4}{*}{ Present study } & None & & 3 & $8 \cdot 1$ & $2 \cdot 1$ & $1 \cdot 5$ & $0 \cdot 7$ \\
\hline & Rheumatoid arthritis & $<35$ years & 4 & $6 \cdot 8$ & $2 \cdot 9$ & 1.9 & 0.6 \\
\hline & Osteoarthritis & & 3 & 4 & 2 & $1 \cdot 4$ & $0 \cdot 4$ \\
\hline & Rheumatoid arthritis & $>40$ years & & $2 \cdot 1$ & $1 \cdot 2$ & 0.9 & $0 \cdot 4$ \\
\hline \multirow{3}{*}{$\begin{array}{c}\text { Urayama } \\
(1956)\end{array}$} & None & & 28 & 2 & $1 \cdot 4$ & $1 \cdot 2$ & $0 \cdot 6$ \\
\hline & Rheumatoid arthritis & $<35$ years & & $1 \cdot 2$ & $0 \cdot 8$ & $0 \cdot 8$ & 0.5 \\
\hline & Rheumatoid arthritis & $>40$ years & 8 & 2 & $1 \cdot 2$ & 0.7 & $0 \cdot 3$ \\
\hline \multirow{2}{*}{$\begin{array}{l}\text { Jayson and Dixon } \\
(1970 a)\end{array}$} & None & & 12 & 5 & & & 0.7 \\
\hline & Rheumatoid arthritis & & 14 & 1 & & & 0.5 \\
\hline
\end{tabular}

to try to relate the observed effects of distension in these joints with the mechanisms probably responsible for the pressure-volume relationships within a joint. Capsular compliance is a 
measure of the ease with which the soft-tissue integument surrounding the joint cavity may be distended. The integument consists mainly of the capsule and its associated ligaments. As saline is instilled into the joint, there is an initial phase of passive filling of the joint cavity (Caughey and Bywaters 1963). During this first phase the compliance is high (the pressure rises slowly). After a certain critical volume is reached, the second period begins in which the capsule and ligaments begin to resist the distending force of the fluid. In this period the compliance decreases rapidly as the integument is stretched more and more. Finally, the elastic limit of the capsule and ligaments strongly resist further distension, and a slight increase in volume results in a large pressure increase-that is, the compliance has approached a minimum which in normal joints was found to have a value of approximately 0.7 millilitre per millimetre of mercury. Low pressure compliance thus depends largely on a critical joint volume (cavity size) and high pressure compliance on the resistance of the integument to internal distending forces.

There was good agreement between the high pressure compliance values obtained for normal human knee joints in our study and those values estimated from findings presented by Urayama (1956) and by Jayson and Dixon $(1970 a, b, c)$ (Table 11); but the values for low pressure compliance estimated from Urayama's results (1956) were much lower (Table II) than were those estimated from the results of Jayson and Dixon $(1970 a, b, c)$ or from our own observations. This difference in compliance must almost certainly reflect some structural differences in the joints of European and Japanese subjects.

Our results in younger patients indicate that rheumatoid arthritis alone produces only a small reduction in capsular compliance. Likewise ageing alone (elderly patients with osteoarthritis) seems to result in a similar modest fall in compliance, seen principally at low intra-articular pressures. The quite substantial fall in capsular compliance seen in the older patients with rheumatoid disease, however, indicates a combination of the effects of both age and disease (Table II). This change presumably reflected a reduction in cavity size due to soft-tissue swelling, synovial proliferation and fibrosis.

Urayama's results (1956), which could be related to age, were in agreement with our own, in that reduction in compliance was greater in older patients with rheumatoid disease, at least at intra-articular pressures above 15 millimetres of mercury. This effect could also be deduced from Jayson and Dixon's results (1970a) for, although their findings were not analysed according to age, their patients with rheumatoid arthritis were nevertheless described as "mainly small, female, middle-aged or elderly". It must be admitted, however, that our results have the same limitations noted by Jayson and Dixon (1970b): that it was not possible to match our normal and patient groups for age, body-build and sex.

The observation that the pressure-volume curves obtained by withdrawal of fluid were usually displaced in the direction of greater compliance compared with those obtained by fluid injection was interpreted as indicating a hysteresis phenomenon of the joint tissues. That is, a given change in volume produced a greater pressure change when the distending force was being removed than when it was being applied. Such a phenomenon would suggest that joint tissues tend to be deformed while under tension and that recovery from this deformation takes time.

This distension deformation was also suggested by the results of the pressure-time studies (Fig. 3), for any loss in fluid volume over the two-hour period was not large enough to account for the associated drop in pressure. Deformation involving elastic and possibly some inelastic stretching of the capsule and ligaments must have occurred. Urayama (1956) found a diminution of intra-articular pressure in relationship to intra-articular volume on repeatedly instilling and withdrawing saline from the joint cavity, which seems to have reflected the same phenomenon. It is evident that further studies of capsular compliance and pressure-volume relationships in normal and diseased joints will lead to a better understanding of normal joint function on the one hand and of the mechanism and effects of joint damage on the other.

VOL. 54 B, NO. 4, NOVEMBER 1972 


\section{SUMMARY}

1. Joint compliance estimations were made on the knee joints of fourteen patients with rheumatoid arthritis, three patients with osteoarthritis and three healthy volunteers.

2. Diminishing values for capsular compliance were found in the normal volunteers, the patients under the age of thirty-five years with rheumatoid arthritis, the patients with osteoarthritis and the patients over the age of forty years with rheumatoid arthritis respectively. 3 . In the young adult normal volunteers the average compliance was 8.1 cubic centimetres per millimetre of mercury at an intra-articular pressure of 5 millimetres of mercury and 0.7 cubic centimetre per millimetre of mercury at 50 millimetres of mercury. In the older patients with rheumatoid disease the average compliance was $2 \cdot 1$ cubic centimetres per millimetre of mercury at 5 millimetres of mercury and 0.4 cubic centimetre per millimetre of mercury at 50 millimetres of mercury.

4. Both the effects of ageing and the effects of disease appeared to be responsible for the low capsular compliance observed in patients over the age of forty years with rheumatoid arthritis, but duration of disease did not seem to be a contributing factor.

This work was supported in part by a grant from the Golden Kiwi Medical Research Distribution Committee and in part by the Medical Research Council of New Zealand. The technical assistance of Mrs R. E. Kenny is gratefully acknowledged.

\section{REFERENCES}

Caughey, D. E., and Bywaters, E. G. L. (1963): Joint Fluid Pressure in Chronic Knee Effusions. Anmals of the Rheumatic Diseases, 22, 106.

Jayson, M. I. V., and Dixon, A. St J. (1970a): Intra-articular Pressure in Rheumatoid Arthritis of the Knee. I. Pressure Changes during Passive Joint Distension. Annals of the Rheumatic Diseases, 29, 261.

Jayson, M. I. V., and Dixon, A. St J. (1970b): Intra-articular Pressure in Rheumatoid Arthritis of the Knee. II. Effect of Intra-articular Pressure on Blood Circulation to the Synovium. Annals of the Rheumatic Diseases, 29, 266.

Jayson, M. I. V., and Dixon, A. St J. (1970c): Intra-articular Pressure in Rheumatoid Arthritis of the Knee. 1II. Pressure Changes during Joint Use. Annals of the Rheumatic Diseases, 29, 401.

Myers, D. B., and Palmer, D. G. (1970): Joint Compliance (abstract). New Zealand Medical Journal, $71,306$.

Palmer, D. G., and Myers, D. B. (1968): Some Observations of Joint Effusions. Arthritis and Rheumatism, $11,745$.

Ropes, M. W. (1959): Diagnostic Criteria for Rheumatoid Arthritis, 1958 revision. Annals of the Rheumatic Diseases, 18, 49.

Urayama, H. (1956): A Study on the Intra-articular Pressure. Journal of the Japanese Orthopaedic Association, 30, 213. 